\section{Acting on actin}

THEbrush border of the intestine is made up of finger-like protrusions, the microvilli, each supported by a rigid core of actin bundles. The protein that organizes the actin filaments in this manner is villin. E. Friedrich et al. (J. Cell Sci. 105, 765-775; 1993) have pursued their observation that cultured cells transfected with villin develop microvilli on their surfaces by exposing the cells to cytochalasin to block actin filament ends. This causes the microvilli to vanish and short actin rods to materialize in the cytoplasm. Taking the cytochalasin away again leads to the reappearance of the microvilli. So it seems that short actin filaments sit on the membrane, waiting to nucleate the microvillus actin cores-another striking example of the multifarious morphological manifestations of the actin molecule.

\section{Friend or foe?}

GLOBAL warming and ozone depletion are hopelessly entangled in the minds of many government ministers, but given that ozone and its man-made foes the chlorofluorocarbons (CFCs) are also greenhouse gases, they may be excused. A particularly clear head will be needed if considering the policy implications of a report by C. Clerbaux et al. (J. geophys. Res. 98, 10491-10497; 1993). Assessing ten of the hydrohalocarbons being developed as substitutes for the CFCs, the authors warn that although the effects on ozone are indeed less severe, three of the substitutes (known as HCFC22, HCFC142b and HFC125) would trap more infrared radiation, molecule for molecule, than the CFCs they are replacing. Industrial applications may require heavier use of hydrohalocarbons than of CFCs to maintain efficiency, and the problem is likely to linger - these molecules can remain in the atmosphere for decades.

\section{Fishy business}

A YEAR ago, J. M. Burkholder and colleagues reported the discovery of a 'phantom' dinoflagellate responsible, through secretion of a toxin, for the mass death of estuarine fish. E. J. Noga et al. now describe a similar case - a dinoflagellate is again involved, but this time it afflicted fish kept in aquaria (Vet. Rec. 133, 96-97; 1993). The victims were tilapia being acclimatized to brackish water; when they died mysteriously, Noga et al. found a hitherto unknown dinoflagellate was present in the water in large numbers. Experiments with more hapless tilapia showed that the dinoflagellates were stimulated to bloom by the presence of living fish, and that the lethal agent was a filterable toxin. More of these cryptic killers no doubt await identification, but that will probably require more sensitive detection techniques. greater immune response, including that of cytotoxic T cells, might also be achieved if the product was used to boost the immune response in humans already primed with a recombinant avipox virus vector such as canarypox (ref. 7; see table); such a vector should be safe, even in immunocompromised recipients.

Although much more remains to be achieved (including a formulation which would induce strong, persisting mucosal responses, especially in the female reproductive tract), the publication of these results is timely. Similar candidate vaccines are already in clinical trials in HIVinfected individuals and are scheduled to be used in trials on HIV-infected mothers and children starting this year ${ }^{8}$. Plans are also underway to initiate efficacy studies in uninfected groups that have a high risk of infection. The World Health Organization has identified sites for such trials in four developing countries.

As well as sponsoring most of the trials listed in the table, the Division of AIDS at the National Institute of Allergy and Infectious Diseases, Washington, recently established a working group to promote the development and testing of suitable candidate vaccines in phase III efficacy trials. There have been doubts that suitable candidate vaccines would become available in time, but the results of the phase I trial of the Genentech product suggest that a product of this type will be one such candidate.

Gordon Ada is in the Division of Cell Biology, John Curtin School of Medical Research, Australian National University, Canberra, ACT, 2601.

\footnotetext{
1. Sabin, A. B. Proc. natn. Acad. Sci. U.S.A. 89, 8852-8855 (1992)

. Schwartz, D. H. et al. Lancet 342, 69-73 (1993)

3. Haigwood, N. L et al. J. Virol. 66, 172-182 (1992)

4. Ada, G. L. in Fundamental Immunology 3rd edn (ed. Paul,

Ada, G. L. in Fundamental Immunology 3rd edn
W. E.) 1309-1352 (Raven, New York, 1993).

5. Cooney, E. L. etal. Lancet 337, 567-572 (1991)

6. Graham, B. S. et al. J. infect. Dis. 166. 244-252 (1992).

7. Cadoz, M. et al. Lancet 339, 1429-1432 (1992)

8. Cohen, J. Science 258, 1568-1570 (1992)
}

\title{
A nearby protogalaxy?
}

\section{S. van den Bergh}

OUR neighbours are an elderly bunch if, that is, we are talking about galaxies. The 27 known members of the Local Group of galaxies (a loose association about 2 megaparsecs across) have travelled far down the evolutionary pathway; all but one have transformed more than 90 per cent of their baryonic mass from gas to stars. To watch the early stages of galaxy formation, we must look further afield. Now it seems that a young protogalaxy - one that has not yet started to form massive stars - may have been found in the relatively nearby M81 group of galaxies, only 3-3.5 megaparsecs away.

Last year, using the 30-metre IRAM telescope, Brouillet, Henkel and Baudry ${ }^{1}$ tentatively identified a small intergalactic cloud on the eastern outskirts of M81 that was emitting radiation characteristic of carbon monoxide. Together with colleagues $^{2}$, they have now filled in the missing optical data and can confidently assert that the molecular cloud and its environs show no sign of massive star formation.

\section{Tracer}

Why the importance of carbon monoxide? The expected life history of a galaxy is a progression from atomic hydrogen or heavier elements recycled from older stars, through denser condensations of molecular species, in particular $\mathrm{H}_{2}$, to the formation of new stars. Carbon monoxide is an excellent tracer for molecular hydrogen, which is otherwise difficult to detect.

Radio observations in the $21-\mathrm{cm}$ line of neutral atomic hydrogen ${ }^{3}$ show that the $\mathrm{CO}$ cloud is embedded in one of two massive atomic hydrogen ( $\mathrm{H}$ I) complexes in the outer part of M81. The gas complexes do not seem to be directly associated with the spiral arms of this galaxy. It seems likely that they have been formed as a result of recent tidal interactions between M81 (NGC3031), M82 (NGC3034) and $\mathrm{NGC} 3077^{4-7}$, a threefold tug which may have disrupted the disk of M82 (ref. 8 ). It has been estimated ${ }^{4}$ that a quarter of all the hydrogen in the M81 group at present lies in tail and tidal structures outside the optical radii of the main cluster galaxies.

Dwarf galaxies might, it is thought, form tidal tails such as these ${ }^{9-11}$. Henkel $e t$ $a{ }^{2}{ }^{2}$ propose that their $\mathrm{CO}$ cloud is a protogalaxy that represents the missing link between tidal $\mathrm{H} \mathrm{I}$ arms and active star-forming regions, such as that lying at the tip of one of the tidal arms in the interacting galaxies NGC4038 and 4039 (the Antennae).

What might happen next? An infant dwarf galaxy formed from a tidal tail might differ from more ancient dwarfs in several important ways ${ }^{12}$. First, tidal arms are not expected to entrain much dark matter (because it is too 'hot'; that is, has too large a velocity dispersion to be trapped) so that young dwarfs formed in this way should have low mass-to-light ratios. 